KCL-MTH-02-02

hep-th/0201113

\title{
D-branes from conformal field theory
}

\author{
Matthias R. Gaberdiel \\ Department of Mathematics \\ King's College London \\ Strand \\ London WC2R 2LS \\ United Kingdom \\ mrg@mth.kcl.ac.uk
}

\begin{abstract}
An introduction to the construction of D-branes using conformal field theory methods is given. A number of examples are discussed in detail, in particular the construction of all conformal D-branes for the theory of a single free boson on a circle.
\end{abstract}

\section{Introduction}

D-branes in string theory can be described and analysed in essentially two different ways. First, one can think of D-branes as being extended objects in space-time that can wrap around certain cycles in the target space geometry. From this point of view, D-branes are described by geometrical data such as cohomology and K-theory [1, 2, 3]. The second point of view, on the other hand, is not geometrical at all: D-branes correspond to (additional) open string sectors that can be added consistently to a given (closed) string theory. In this 'world-sheet' approach, D-branes are described by (boundary) conformal field theory.

The boundary conformal field theory description is an exact string theory description, but it is often only available at specific points in the moduli space of target space geometries, such as orbifold points [1, 5, 6, 7, 8, 9, 10, 11, 12], Gepner points in Calabi-Yau manifolds [13, 14, 15], etc. On the other hand, the geometrical approach is generically available, but it can only be trusted whenever we are in a regime where the supergravity approximation is good. The two approaches are therefore in some sense complementary, and one can learn interesting features about 'stringy geometry' by comparing their results (see for example [16] and the lectures of Wolfgang Lerche at this school).

In this lecture I shall attempt to give a pedagogical introduction to the conformal field theory approach. I shall also explain some recent work (in collaboration with Andreas Recknagel and Gérard Watts [17, 18]) that suggests that the conformal field theory approach is tractable for larger classes of theories than had hitherto be thought. 


\section{The boundary conformal field theory approach}

Suppose we are given a conformal field theory defined on closed Riemann surfaces, i.e. a closed string theory. The main question we want to address is: how can we extend this conformal field theory to a theory that is also defined on world-sheets with boundary. More precisely we want to ask which boundary conditions can be imposed at the various boundaries. From a string theory point of view, this is the question of which open strings can be consistently added to a given closed string theory.

In some sense, this problem is rather similar to a familiar construction in (closed) conformal field theory. Suppose we are given the theory defined on the sphere. We can then ask whether this theory determines already (uniquely) the theory on arbitrary Riemann surfaces. The answer is well known [19, 20, 21]: the theory on the sphere determines uniquely the theory on an arbitrary closed Riemann surface (if it exists), but it does not guarantee that it is consistent. Indeed, there is one additional consistency condition that arises at genus 1 (and that does not follow from the consistency of the theory on the sphere), namely that the correlation functions on the torus transform under the action of the modular group $\operatorname{SL}(2, \mathbb{Z})$. If this consistency condition is satisfied, the theory is consistent on all Riemann surfaces [21].

The analogous result for the construction of the theory on surfaces with boundaries is not really known. For a given theory defined on the sphere, the complete list of 'sewing relations' that have to be satisfied by each boundary condition is known [22, 23]. However it is not clear for which classes of theories solutions to these sewing relations can be found, and if so, how many. Based on the examples that have been understood [24, 25, 26, 27, 28, 29 it appears that modular invariance may again be sufficient to guarantee that a 'complete' set of boundary conditions can be constructed. In fact, there are striking similarities between the classification of modular invariant partition functions and that of the so-called NIM-reps (non-negative integer matrix representations of the fusion algebra) that appear naturally in the construction of the boundary states [26, 30]. On the other hand, it seems that there are more NIM-reps than (consistent) conformal field theories that can be defined on the torus, and at least some of the additional NIM-reps seem to be naturally related to consistent conformal field theories that are only defined on the sphere (but not on the torus).

The basic reason why the theory on the sphere determines already the theory on all Riemann surfaces can be schematically understood as follows. Since we are dealing with a local conformal field theory, the operator product expansion of any two operators is the same, irrespective of the surrounding surface. The operator product expansion (and thus the 'local structure' of the theory) is therefore already determined by the theory on the sphere.

For the problem we are actually interested in, namely the extension of the theory on the sphere, say, to surfaces with boundary, a similar consideration applies. As we have just explained, given the theory on the sphere we can deduce the operator product expansion of the fields $\phi_{a}$, which we can write schematically as

$$
\phi_{a} \phi_{b} \sim \sum_{c} C_{a b}^{c} \phi_{c}
$$

Here $C_{a b}^{c}$ are the structure constants of the theory on the sphere, and we have suppressed the dependence of the fields on the coordinates on the sphere. We can think of the

\footnotetext{
${ }^{1}$ For example, the theory of a single NS fermion is consistent on the sphere but does not satisfy the modular consistency condition.
} 
operator product expansion as defining an 'algebra of fields'. Th The boundary conditions we are interested in have to respect this algebra, and they must therefore define an 'algebra homomorphism'

$$
\text { ('algebra of fields') } \longrightarrow \mathbb{C} \text {. }
$$

Every element of the space of states of the theory on the sphere $\mathcal{H}$ defines a map of the form (2), and in fact every such map arises from a suitable (infinite) linear combination of such states. Thus we can describe each boundary condition by a 'coherent' boundary state in $\mathcal{H}^{\mathrm{p}}$, and for the boundary condition labelled by $\alpha$ we denote the corresponding boundary state by $\| \alpha\rangle\rangle$. Given this boundary state, the amplitudes of the fields in the presence of the boundary with boundary condition $\alpha$ are then simply given by the (closed string) expression

$$
\left.\left\langle\phi_{1} \phi_{2} \phi_{3}\right\rangle_{\alpha}=\left\langle\phi_{1} \phi_{2} \phi_{3} \| \alpha\right\rangle\right\rangle
$$

Not every linear map of the form (2) actually defines a boundary state. (Indeed, it follows from the above discussion, that there exists for example a coherent state for each higher genus Riemann surface.) The coherent states that describe boundary conditions are characterised by the property that the left- and right-moving fields corresponding to unbroken symmetries are related to one another at the boundary. If we take the boundary to be along the real axis, the relevant condition is that

$$
S(z)=\rho(\bar{S}(\bar{z})) \quad \text { for } z \in \mathbb{R},
$$

where $S$ and $\bar{S}$ are generators of the symmetry that is preserved by the boundary, and $\rho$ denotes an automorphism of the algebra of fields that leaves the stress-energy tensor invariant. The fields $S(z)$ and $\bar{S}(\bar{z})$ have an expansion in terms of modes as

$$
S(z)=\sum_{n \in \mathbb{Z}} S_{n} z^{-n-h}, \quad \bar{S}(\bar{z})=\sum_{n \in \mathbb{Z}} \bar{S}_{n} \bar{z}^{-n-h},
$$

where $h$ is the conformal weight of $S$ (and $\bar{S}$ ). In the description of the boundary condition in terms of a boundary state, the boundary is taken to be the (unit) circle around the origin. In order to express the above condition in terms of a condition involving the boundary state, we apply the conformal transformations $\zeta(z)=e^{-2 \pi \imath z}$ and $\bar{\zeta}(\bar{z})=e^{+2 \pi \imath \bar{z}}$ to obtain

$$
(-2 \pi \imath \zeta)^{h} S(\zeta)=(2 \pi \imath \bar{\zeta})^{h} \rho(\bar{S}(\bar{\zeta})) \quad \text { for }|\zeta|=1,
$$

where we have used that a (primary) conformal field transforms as

$$
S(z) \mapsto \zeta^{\prime}(z)^{h} S(\zeta(z))
$$

and similarly for $\bar{S}$. Using (5) we thus find that a boundary state $\| \alpha\rangle\rangle$ that preserves the symmetry described by $S$ has to satisfy

$$
\left.\left.\left(\sum_{n \in \mathbb{Z}} S_{n} \zeta^{-n}-(-1)^{h} \sum_{n \in \mathbb{Z}} \rho\left(\bar{S}_{n}\right) \bar{\zeta}^{-n}\right) \| \alpha\right\rangle\right\rangle=0 \quad \text { for }|\zeta|=1 .
$$

\footnotetext{
${ }^{2}$ Because of the dependence on the coordinates, this is not really an algebra, but rather (a slight generalisation of) what is usually called a vertex operator algebra.

${ }^{3}$ As we shall see momentarily, the boundary states are necessarily coherent states, i.e. they do not lie in the Fock space of finite energy states.
} 
Since this has to hold for all $\zeta$ with $|\zeta|=1$, (8) implies the so-called 'gluing condition'

$$
\left.\left.\left(S_{n}-(-1)^{h} \rho\left(\bar{S}_{-n}\right)\right) \| \alpha\right\rangle\right\rangle=0 \quad \text { for all } n \in \mathbb{Z} .
$$

The gluing condition implies in particular that $\| \alpha\rangle\rangle$ must be a coherent state since no vector in the Fock space of finite energy states can satisfy (9) for all $n \in \mathbb{Z}$.

There is only one symmetry that every boundary condition has to preserve. This is the 'conformal' symmetry that guarantees that the resulting field theory is again conformal. In terms of (9) it corresponds to the gluing condition

$$
\left.\left.\left(L_{n}-\bar{L}_{-n}\right) \| \alpha\right\rangle\right\rangle=0 \quad \text { for all } n \in \mathbb{Z},
$$

where $L_{n}$ and $\bar{L}_{n}$ are the modes of the left- and right-moving stress energy tensor of conformal weight $h_{L}=h_{\bar{L}}=2$. If we impose other gluing conditions beyond (10) we are restricting our attention to special boundary conditions. However, from an abstract point of view, one would expect that all conformal boundary conditions will play a rôle.

The more symmetries we require the boundary condition to preserve, the fewer boundary conditions exist, and the more tractable the problem becomes. The situation is particularly simple if the closed theory is a 'rational' theory with respect to the preserved symmetry algebra: let us assume we are interested in boundary conditions that respect the symmetry algebra $\mathcal{A}$ (where we take, for simplicity, $\rho=\mathrm{id}$ ). In order to determine the relevant boundary conditions we decompose the space of states of the closed string theory $\mathcal{H}$ in terms of representations of $\mathcal{A} \otimes \overline{\mathcal{A}}$ as

$$
\mathcal{H}=\bigoplus_{i, j} N_{i j} \mathcal{H}_{i} \otimes \overline{\mathcal{H}}_{j}
$$

where the sum runs over the set of irreducible representations of $\mathcal{A}$ and $\overline{\mathcal{A}} \cong \mathcal{A}$, and $N_{i j}$ describes the multiplicity with which the irreducible representation $\mathcal{H}_{i} \otimes \overline{\mathcal{H}}_{j}$ of $\mathcal{A} \otimes \overline{\mathcal{A}}$ appears in $\mathcal{H}$. The theory is called 'rational with respect to $\mathcal{A}$ ' if $\mathcal{A}$ only possesses finitely many irreducible representations. In this case, the sum in (11) is finite.

Since the modes that appear in the gluing condition (9) map each $\mathcal{H}_{i} \otimes \overline{\mathcal{H}}_{j}$ into itself, we can solve the gluing constraint separately for each summand in (11). We can find a non-trivial solution provided that $\mathcal{H}_{i}$ is the conjugate representation of $\overline{\mathcal{H}}_{j}$. If this is the case, there is (up to normalisation) only one coherent state that satisfies (9); ; this state is called the Ishibashi state [31] and it is denoted by

$$
\left.|i\rangle\rangle \in \mathcal{H}_{i} \otimes \overline{\mathcal{H}}_{i}, \quad\left(S_{n}-(-1)^{h_{S}} \rho\left(\bar{S}_{-n}\right)\right)|i\rangle\right\rangle=0 \quad \text { for all } n \in \mathbb{Z} \text { and } S \in \mathcal{A} .
$$

If the theory is rational then there are in particular only finitely many Ishibashi states.

Since every boundary state satisfies the gluing condition (9) it must be a linear combination of the Ishibashi states. We can therefore write every boundary state as

$$
\left.\| \alpha\rangle\rangle=\sum_{i} B_{\alpha}^{i}|i\rangle\right\rangle,
$$

where $B_{\alpha}^{i}$ are some constants that characterise the boundary condition. These constants are constrained by two classes of conditions:

(i) The Cardy condition [32].

\footnotetext{
${ }^{4}$ We are assuming here, for ease of notation, that the multiplicities $N_{i j}$ are all either zero or one; the modifications for the general case are obvious.
} 
(ii) The so-called 'sewing relations' that were first derived in [23, 22].

The Cardy condition (i) comes about as follows. Let us consider the (open string) partition function

$$
Z_{\alpha \beta}(\tilde{q})=\operatorname{Tr}_{\mathcal{H}_{\alpha \beta}} e^{-2 \pi T H_{o}}=\sum_{i} n_{\alpha \beta}^{i} \chi_{i}(\tilde{q})
$$

of the open string with boundary conditions $\alpha$ and $\beta$ at the two ends. Here $\mathcal{H}_{\alpha \beta}$ is the corresponding space of open string states, and $H_{o}$ the open string Hamilton operator (that equals $H_{o}=L_{0}-\frac{c}{24}$ ). In writing the second equation in (14) we have used that the boundary conditions preserve $\mathcal{A}$, and therefore that we can decompose $\mathcal{H}_{\alpha \beta}$ with respect to $\mathcal{A}$ as

$$
\mathcal{H}_{\alpha \beta}=\bigoplus_{i} n_{\alpha \beta}^{i} \mathcal{H}_{i}
$$

where each $\mathcal{H}_{i}$ is an irreducible representation of $\mathcal{A}$. The numbers $n_{\alpha \beta}^{i}$ describe the multiplicity with which $\mathcal{H}_{i}$ appears in $\mathcal{H}_{\alpha \beta}$, and they are therefore non-negative integers. (In fact, the numbers $n_{\alpha \beta}^{i}$ are precisely the entries of the NIM-reps we mentioned before.) We have furthermore used the usual short hand notation for the character of a representation,

$$
\chi_{i}(\tilde{q})=\operatorname{Tr}_{\mathcal{H}_{i}}\left(e^{-2 \pi T\left(L_{0}-\frac{c}{24}\right)}\right), \quad \tilde{q}=e^{-2 \pi T} .
$$

In terms of the boundary states we introduced before (i.e. from the closed string point of view) this amplitude is simply the overlap

$$
\left\langle\left\langle\alpha\left\|e^{-2 \pi L H_{c l}}\right\| \beta\right\rangle\right\rangle=\sum_{i}\left(B_{\alpha}^{i}\right)^{*} B_{\beta}^{i} \chi_{i}(q) .
$$

Here $H_{c l}$ is the closed string Hamiltonian, and we have used (13) to write the boundary states in terms of the Ishibashi states. We have furthermore used that

$$
\left\langle\left\langle i\left|e^{-2 \pi L H_{c l}}\right| j\right\rangle\right\rangle=\delta_{i j} \chi_{i}(q),
$$

where $\chi_{i}(q)$ is again the character of the representation $\mathcal{H}_{i}$ that is now evaluated at $q$ with $q=e^{-2 \pi L}$ rather than $\tilde{q}$. In relating the open- and closed point of view, i.e. in relating (14) and (17), we have to exchange what we think of as being the time- and space-coordinate on the world-sheet; we therefore have to identify $T=1 / L$. If we write

$$
q=e^{2 \pi i \tau}, \quad \tau=i L
$$

then $\tilde{q}$ is simply given as

$$
\tilde{q}=e^{-\frac{2 \pi i}{\tau}} .
$$

Thus $q$ and $\tilde{q}$ are related by the standard modular $S$-transformation that maps $\tau \mapsto-1 / \tau$. At least for rational conformal field theories (and in fact under certain slightly weaker conditions) the characters of the irreducible representations transform into one another as

$$
\chi_{i}(q)=\sum_{j} S_{j i} \chi_{j}(\tilde{q}),
$$

where $S_{i j}$ is the symmetric and unitary matrix representing the $S$-transformation of the modular group $\mathrm{SL}(2, \mathbb{Z})$. Inserting (21) into (17) we therefore find that

$$
Z_{\alpha \beta}(\tilde{q})=\sum_{i, j}\left(B_{\alpha}^{i}\right)^{*} B_{\beta}^{i} S_{j i} \chi_{j}(\tilde{q})
$$


Comparing with (14), and assuming that the characters of the irreducible representations are linearly independent, it therefore follows that

$$
n_{\alpha \beta}^{j}=\sum_{i}\left(B_{\alpha}^{i}\right)^{*} B_{\beta}^{i} S_{j i}
$$

This is a very restrictive condition that is often (in particular, if the theory is rational and there are only finitely many irreducible representations) fairly accessible.

Before proceeding we should note that the set of solutions to Cardy's condition form (the positive cone of) a lattice: suppose that the set

$$
\left.\left.\left.\left.M=\left\{\| \alpha_{1}\right\rangle\right\rangle, \ldots, \| \alpha_{n}\right\rangle\right\rangle\right\}
$$

satisfies Cardy's condition, i.e. the overlap between any two elements of $M$ leads to nonnegative integer numbers $n_{\alpha_{j}, \alpha_{k}}^{i}$, then so does the set

$$
\left.\left.\left.\left.\left.\left.M^{\prime}=\left\{\| \alpha_{1}\right\rangle\right\rangle, \ldots, \| \alpha_{n}\right\rangle\right\rangle, \sum_{l=1}^{n} m_{l} \| \alpha_{l}\right\rangle\right\rangle\right\},
$$

provided that $m_{l} \in \mathbb{N}_{0}$ for $l=1, \ldots, n$. This is simply a consequence of the fact that sums of products of non-negative integers are non-negative integers. What we therefore want to find are the fundamental boundary conditions that generate all other boundary conditions upon taking positive integer linear combinations as above.

In general, rather little is known about how to characterise these fundamental D-branes intrinsically. Part of the problem is due to the fact that the Cardy condition by itself does not have a unique solution. In order to illustrate this fact let us consider one of the simplest conformal field theories, the so-called Ising model. This theory is the minimal Virasoro model with $c=\frac{1}{2}$ for which

$$
\mathcal{H}=\left(\mathcal{H}_{0} \otimes \overline{\mathcal{H}}_{0}\right) \bigoplus\left(\mathcal{H}_{\frac{1}{2}} \otimes \overline{\mathcal{H}}_{\frac{1}{2}}\right) \bigoplus\left(\mathcal{H}_{\frac{1}{16}} \otimes \overline{\mathcal{H}}_{\frac{1}{16}}\right)
$$

Here $\mathcal{H}_{h}$ is the irreducible representation of the Virasoro algebra for which the highest weight state has $L_{0}$ eigenvalue equal to $h$. In the basis corresponding to $\mathcal{H}_{0}, \mathcal{H}_{\frac{1}{2}}, \mathcal{H}_{\frac{1}{16}}$, the $S$-matrix is given as

$$
S=\frac{1}{2}\left(\begin{array}{ccc}
1 & 1 & \sqrt{2} \\
1 & 1 & -\sqrt{2} \\
\sqrt{2} & -\sqrt{2} & 0
\end{array}\right) .
$$

One set of (fundamental) boundary states can be constructed following the general procedure of Cardy [32 as follows. For each irreducible representation $\mathcal{H}_{i}$ we define the boundary state $\left.\left.\| \alpha_{i}\right\rangle\right\rangle$ in terms of the Ishibashi states by

$$
\left.\left.\left.\| \alpha_{j}\right\rangle\right\rangle=\sum_{l} \frac{S_{j l}}{\sqrt{S_{0 l}}}|l\rangle\right\rangle .
$$

For these boundary states, the corresponding integers $n_{\alpha_{j} \alpha_{k}}^{i}$ are then simply the fusion matrices, since (23) becomes in this case

$$
n_{\alpha_{j} \alpha_{k}}^{i}=\sum_{l} \frac{S_{j l}^{*}}{\sqrt{S_{0 l}}} \frac{S_{k l}}{\sqrt{S_{0 l}}} S_{i l}=\sum_{l} \frac{S_{i l} S_{k l} S_{j l}^{*}}{S_{0 l}},
$$

which equals the fusion matrix $N_{i k}^{j}$ by Verlinde's formula 33. 
Applying the general formula to the case at hand, the three Cardy boundary conditions are then

$$
\begin{aligned}
\left.\left.\| \alpha_{0}\right\rangle\right\rangle & \left.\left.\left.=\frac{1}{\sqrt{2}}|0\rangle\right\rangle+\frac{1}{\sqrt{2}}\left|\frac{1}{2}\right\rangle\right\rangle+\frac{1}{2^{\frac{1}{4}}}\left|\frac{1}{16}\right\rangle\right\rangle, \\
\left.\left.\| \alpha_{\frac{1}{2}}\right\rangle\right\rangle & \left.\left.\left.=\frac{1}{\sqrt{2}}|0\rangle\right\rangle+\frac{1}{\sqrt{2}}\left|\frac{1}{2}\right\rangle\right\rangle-\frac{1}{2^{\frac{1}{4}}}\left|\frac{1}{16}\right\rangle\right\rangle, \\
\left.\left.\| \alpha_{\frac{1}{16}}\right\rangle\right\rangle & \left.=|0\rangle\rangle-\left|\frac{1}{2}\right\rangle\right\rangle .
\end{aligned}
$$

However, this does not define the only solution to Cardy's conditions. Indeed, another set of 'fundamental' boundary states is given by the three boundary states

$$
\begin{aligned}
&\| 1\rangle\rangle\left.\left.=|0\rangle\rangle+\left|\frac{1}{2}\right\rangle\right\rangle+2^{\frac{1}{4}}\left|\frac{1}{16}\right\rangle\right\rangle, \\
&\left.\| 2\rangle\rangle=|0\rangle\rangle+\left|\frac{1}{2}\right\rangle\right\rangle, \\
&\left.\| 3\rangle\rangle=\sqrt{2}|0\rangle\rangle-\sqrt{2}\left|\frac{1}{2}\right\rangle\right\rangle .
\end{aligned}
$$

It is not difficult to check that these three boundary states also satisfy Cardy's condition, i.e. that the relative overlaps give rise to non-negative integers (23). However, these two sets of boundary states are mutually incompatible since, for example, the overlap

$$
\left\langle\left\langle\alpha_{0}\left\|e^{-2 \pi L H_{c l}}\right\| 1\right\rangle\right\rangle=\sqrt{2} \chi_{0}(\tilde{q}) .
$$

While both of these sets of boundary states satisfy Cardy's condition, it is believed that only (30) actually defines consistent boundary states, and that the other consistency conditions (some of which we are about to discuss) will rule out (31). However, this example demonstrates that Cardy's consistency condition alone does not allow one to discard (31).

Suppose now that we have found a solution to Cardy's condition that also satisfies all other consistency conditions. We would like to find a good criterion that allows us to find the fundamental boundary states out of which all other boundary states can be obtained as positive integer linear combinations. As we mentioned before, little is known about this in general, but it is believed that the fundamental boundary states are characterised by the condition that the self-overlap of each such boundary state $\| \alpha\rangle\rangle$ contains the vacuum representation in the open string channel with multiplicity one, i.e.

$$
\left\langle\left\langle\alpha\left\|e^{-2 \pi L H_{c l}}\right\| \alpha\right\rangle\right\rangle=\chi_{0}(\tilde{q})+\cdots .
$$

With this criterion any non-trivial integer linear combination of fundamental boundary states is then not fundamental. Incidentally, according to this criterion the three Cardy states are fundamental, but for example $\| 1\rangle\rangle$ is not. In the following we shall call a family of boundary states fundamental if (33) is satisfied for all its members.

For D-branes that are fundamental in this sense, one of the sewing relations simplifies considerably, and actually gives rise to a powerful constraint (see [17 for a more detailed derivation). This constraint arises from considering a two-point function of primary fields in the presence of such a boundary condition, 


$$
\begin{aligned}
& \varphi_{a}(z, \bar{z}) \\
& \varphi_{b}(w, \bar{w}) \\
& F_{a b}(z, \bar{z}, w, \bar{w})=\left\langle\varphi_{a}(z, \bar{z}) \varphi_{b}(w, \bar{w})\right\rangle .
\end{aligned}
$$

The gluing conditions for the energy-momentum tensor imply that (34) can be described in terms of four-point chiral blocks where we insert chiral vertex operators of weight $h_{a}, \bar{h}_{a}, h_{b}$ and $\bar{h}_{b}$ at $z, \bar{z}, w$ and $\bar{w}$, respectively. This four-point function can then be factorised in two different ways, leading to two different representations of the correlation function, as shown below. In the first picture one considers the limit in which the two fields approach the boundary separately; in the second picture on the other hand, the two fields come close together away from the boundary, and we can thus use the operator product expansion (1) in order to express the product of these two fields in terms of a sum of single fields:
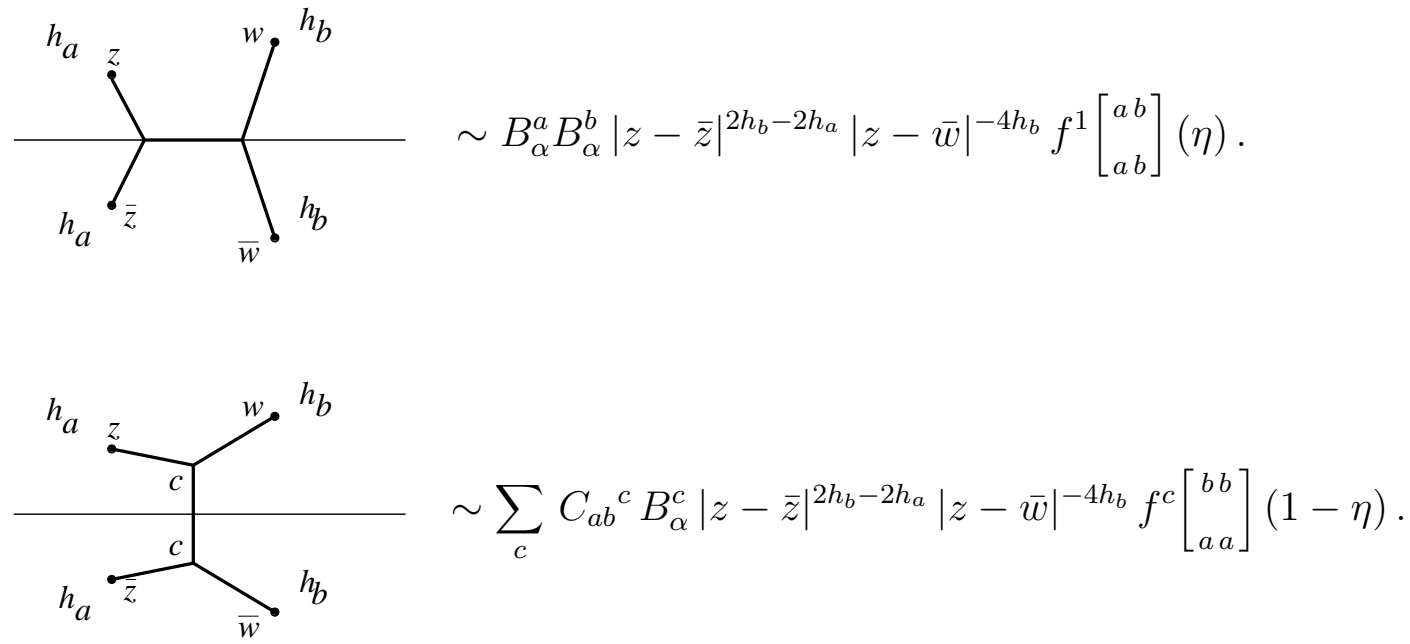

In writing down these equations we have specialised to the case where $\varphi_{a}$ and $\varphi_{b}$ are self-conjugate fields for which $h_{a}=\bar{h}_{a}$ and $h_{b}=\bar{h}_{b}$. The $f^{1}$ and $f^{c}$ denote the different chiral four-point blocks, and $\eta$ is the cross-ratio $\eta=|(z-w) /(z-\bar{w})|^{2}$ which is real with $0 \leq \eta \leq 1$. In both equations we have only considered the leading behaviour as $\eta \rightarrow 1$, i.e. we have only taken into consideration the contribution of the vacuum state in the open string channel. (This is where we have used the assumption that the boundary condition in question is fundamental to deduce that there is only one such state.)

The two sets of chiral blocks are related by the so-called fusing matrices

$$
f^{c}\left[\begin{array}{c}
b b \\
a a
\end{array}\right](1-\eta)=F_{c 1}\left[\begin{array}{c}
b b \\
a a
\end{array}\right] f^{1}\left[\begin{array}{l}
a b \\
a b
\end{array}\right](\eta) .
$$

Substituting (37) in (36) and comparing with (35), we then obtain the sewing relation

$$
B_{\alpha}^{a} B_{\alpha}^{b}=\sum_{c} C_{a b}^{c} F_{c 1}\left[\begin{array}{c}
b b \\
a a
\end{array}\right] B_{\alpha}^{c} .
$$

This condition is known as the 'factorisation constraint' [23, 22], the 'cluster condition' [34] or the 'classifying algebra' [35]. In many cases it is, however, rather difficult to check since the structure constants $C$ and $F$ on the right hand side are often not explicitly known. However, there are a few examples where one can actually determine the structure of (38) explicitly, and use this to classify all possible fundamental D-branes of a theory. We shall come back to this point later. 


\section{Some illustrative examples}

In this section we want to give some examples that will illustrate the structure we have described above.

\subsection{A free boson on a circle of radius $R$}

This theory is generated by the modes of the field $X(z, \bar{z})$ for which we identify $X$ with $X+2 \pi R$. The left- and right-moving modes are denoted by $\alpha_{l}$ and $\bar{\alpha}_{l}$, respectively, and they satisfy the commutation relations

$$
\begin{aligned}
{\left[\alpha_{l}, \alpha_{k}\right] } & =l \delta_{l,-k} \\
{\left[\alpha_{l}, \bar{\alpha}_{k}\right] } & =0 \\
{\left[\bar{\alpha}_{l}, \bar{\alpha}_{k}\right] } & =l \delta_{l,-k} .
\end{aligned}
$$

The space of states of the theory is

$$
\mathcal{H}=\bigoplus_{m, n} \mathcal{H}_{(m, n)}
$$

where $\mathcal{H}_{(m, n)}$ consists of the states that are generated by the action of the negative modes $\alpha_{-l}$ and $\bar{\alpha}_{-l}$ with $l>0$ from a ground-state $\left|\left(p_{L}, p_{R}\right)\right\rangle$ for which

$$
\alpha_{0}\left|\left(p_{L}, p_{R}\right)\right\rangle=p_{L}\left|\left(p_{L}, p_{R}\right)\right\rangle \quad \bar{\alpha}_{0}\left|\left(p_{L}, p_{R}\right)\right\rangle=p_{R}\left|\left(p_{L}, p_{R}\right)\right\rangle
$$

with

$$
\left(p_{L}, p_{R}\right)=\left(\frac{m}{2 R}+n R, \frac{m}{2 R}-n R\right) .
$$

The branes that respect the $\mathrm{U}(1)$ symmetry corresponding to $\alpha_{l}$ and $\bar{\alpha}_{l}$ are either $\mathrm{Neu}$ mann $(\mathrm{N})$ or Dirichlet (D) branes. They are characterised by the gluing conditions

$$
\begin{array}{lll}
\left.\left(\alpha_{l}+\bar{\alpha}_{-l}\right)|\mathrm{N}\rangle\right\rangle=0 & l \in \mathbb{Z}, \\
\left.\left(\alpha_{l}-\bar{\alpha}_{-l}\right)|\mathrm{D}\rangle\right\rangle=0 & l \in \mathbb{Z} .
\end{array}
$$

Since $\alpha_{l}$ are the modes of a field of conformal weight one, it follows from (9) that the Neumann gluing condition corresponds to $\rho=\mathrm{id}$, while the automorphism for the Dirichlet gluing condition is $\rho\left(\alpha_{l}\right)=-\alpha_{l}$. This automorphism leaves the stress energy tensor invariant since the Virasoro generators are bilinear (normal ordered) products of the $\alpha_{l}$ modes. It also follows from this fact that both gluing conditions actually imply the conformal gluing condition (10).

The condition (42) with $l=0$ implies that a Neumann Ishibashi state can only be constructed in $\mathcal{H}_{(m, n)}$ provided that $p_{L}=-p_{R}$. In terms of our previous discussion this is simply the statement that the left- and right representations of the preserved symmetry algebra must be conjugate representations. At a generic radius $R, p_{L}=-p_{R}$ can only be satisfied if $m=0$, and thus we have a Neumann Ishibashi state for each $n \in \mathbb{Z}$,

$$
|(n R,-n R)\rangle\rangle^{\mathrm{N}} \in \mathcal{H}_{(0, n)} .
$$

Similarly, a Dirichlet Ishibashi state can only be constructed in $\mathcal{H}_{(m, n)}$ provided that $p_{L}=p_{R}$; at a generic radius we therefore only have the Dirichlet Ishibashi states

$$
\left.\left|\left(\frac{m}{2 R}, \frac{m}{2 R}\right)\right\rangle\right\rangle^{\mathrm{D}} \in \mathcal{H}_{(m, 0)},
$$


where $m \in \mathbb{Z}$. In this simple example, one can actually give a closed formula for these Ishibashi states; they are simply given as

$$
\begin{aligned}
|(n R,-n R)\rangle\rangle^{\mathrm{N}} & =\exp \left(\sum_{l=1}^{\infty}-\frac{1}{l} \alpha_{-l} \bar{\alpha}_{-l}\right)|(n R,-n R)\rangle \\
\left.\left|\left(\frac{m}{2 R}, \frac{m}{2 R}\right)\right\rangle\right\rangle^{\mathrm{D}} & =\exp \left(\sum_{l=1}^{\infty} \frac{1}{l} \alpha_{-l} \bar{\alpha}_{-l}\right)\left|\left(\frac{m}{2 R}, \frac{m}{2 R}\right)\right\rangle .
\end{aligned}
$$

It is easy to see that these states satisfy indeed (42) and (43), respectively.

The actual D-branes (that satisfy Cardy's condition) are given as linear combinations of these Ishibashi states. In the present case, the relevant expressions are

$$
\left.\| w\rangle\rangle=R^{\frac{1}{2}} \sum_{n \in \mathbb{Z}} e^{i w n R}|(n R,-n R)\rangle\right\rangle^{\mathrm{N}},
$$

which describes a Neumann brane with Wilson line $w$, and

$$
\left.\| a\rangle\rangle=\frac{1}{(2 R)^{\frac{1}{2}}} \sum_{m \in \mathbb{Z}} e^{i \frac{m a}{R}}\left|\left(\frac{m}{2 R}, \frac{m}{2 R}\right)\right\rangle\right\rangle^{\mathrm{D}},
$$

which corresponds to a Dirichlet brane at the position $a$. Given the explicit form of the Ishibashi states, it is now straightforward to work out the closed string tree diagram, i.e. the overlap (17), and to check that the corresponding open string loop diagram, i.e. the partition function (14), satisfies the Cardy condition. All of the branes above are fundamental.

For both Neumann and Dirichlet branes one can also analyse the corresponding factorisation constraint. In both cases, the relevant classifying algebra simplifies considerably since the combination of $C$ and $F$ that appears in (38) is essentially trivial. More precisely, if we write the boundary states as

$$
\begin{aligned}
\left.\left.\| B^{N}\right\rangle\right\rangle & \left.=R^{\frac{1}{2}} \sum_{n \in \mathbb{Z}} \hat{B}_{n}^{N}|(n R,-n R)\rangle\right\rangle^{\mathrm{N}} \\
\left.\left.\| B^{D}\right\rangle\right\rangle & \left.=\frac{1}{(2 R)^{\frac{1}{2}}} \sum_{m \in \mathbb{Z}} \hat{B}_{m}^{D}\left|\left(\frac{m}{2 R}, \frac{m}{2 R}\right)\right\rangle\right\rangle^{\mathrm{D}},
\end{aligned}
$$

the factorisation constraint simply becomes

$$
\begin{aligned}
\hat{B}_{n_{1}}^{N} \cdot \hat{B}_{n_{2}}^{N} & =\hat{B}_{n_{1}+n_{2}}^{N} \\
\hat{B}_{m_{1}}^{D} \cdot \hat{B}_{m_{2}}^{D} & =\hat{B}_{m_{1}+m_{2}}^{D} .
\end{aligned}
$$

The most general fundamental U(1)-preserving Neumann and Dirichlet branes are thus given by

$$
\hat{B}_{n}^{N}=e^{i w n R} \quad \hat{B}_{m}^{D}=e^{i \frac{m a}{R}},
$$

and therefore correspond to the branes given above. Strictly speaking, one could also choose $w$ and $a$ to be arbitrary complex (rather than real) numbers. While the resulting branes seem to be consistent from a conformal field theory point of view, they have complex couplings to some of the space-time fields, and are therefore presumably unphysical.

The boundary conditions that we have analysed so far are rather special in that they preserve the full U(1)-symmetry rather than just the conformal symmetry. From an 
abstract point of view, it is not clear why these special branes should account for all the relevant branes of the theory, and it is therefore important to understand what other (conformal) branes exist. In order to answer this question let us first consider the case where the radius of the circle is at the self-dual point, $R=\frac{1}{\sqrt{2}}$. Then the theory is the same as the WZW model corresponding to $\mathrm{SU}(2)$ at level $k=1$.

\subsection{The conformal branes for $\mathrm{SU}(2)$ level one}

Let us begin by recalling some properties of this theory on the sphere. The theory has leftand right-moving currents $J^{a}(z)$ and $\bar{J}^{a}(\bar{z})$ whose modes $J_{n}^{a}$ and $\bar{J}_{n}^{a}$ define two commuting copies of the affine algebra $\widehat{\mathrm{su}}(2)_{1}$. The space of states of the theory on the sphere $\mathcal{H}_{\text {sphere }}$ can therefore be decomposed into highest weight representations of these two algebras.

There are two highest weight representations of the affine algebra $\widehat{\operatorname{su}}(2)$ at level 1 that actually define representations of the conformal field theory (or the corresponding vertex operator algebra): the vacuum representation $\mathcal{H}_{0}$ that is generated from a state transforming in the singlet representations of the horizontal su(2) algebra (spanned by the zero modes $J_{0}^{a}$ ), and the representation $\mathcal{H}_{\frac{1}{2}}$ for which the highest weight states transform in the doublet representation of the horizontal $\mathrm{su}(2)$ algebra. The free boson theory at the self-dual radius we are interested in, has a space of states that is described by

$$
\mathcal{H}_{\text {sphere }}=\left(\mathcal{H}_{0}^{\widehat{\mathrm{su}}(2)} \otimes \overline{\mathcal{H}}_{0}^{\widehat{\widehat{s u}}(2)}\right) \oplus\left(\mathcal{H}_{\frac{1}{2}}^{\widehat{\mathrm{su}}(2)} \otimes \overline{\mathcal{H}}_{\frac{1}{2}}^{\widehat{\mathrm{su}}(2)}\right)
$$

Because of the Sugawara construction, every highest weight representation of $\widehat{\operatorname{su}}(2)_{1}$ also forms a representation of the Virasoro algebra Vir with $c=1$. The generators of the Virasoro algebra commute with the current zero modes, and thus the $\widehat{\operatorname{su}}(2)_{1}$ representations can be decomposed into representations of $\mathrm{su}(2) \oplus V$ ir. If we denote the $(2 j+1)$-dimensional spin $j$ representation of $\mathrm{su}(2)$ by $V^{j}$ and the Virasoro algebra irreducible highest weight representation of weight $h$ by $\mathcal{H}_{h}^{\mathrm{Vir}}$, then we have

$$
\mathcal{H}_{j}^{\widehat{\mathrm{su}}(2)}=\sum_{n=0}^{\infty} V^{(n+j)} \otimes \mathcal{H}_{(n+j)^{2}}^{\mathrm{Vir}} .
$$

It thus follows that the space of states can be decomposed with respect to the algebra $\mathrm{su}(2)_{L} \oplus \mathrm{su}(2)_{R} \oplus \operatorname{Vir}_{L} \oplus \operatorname{Vir}_{R}$ as

$$
\mathcal{H}_{\text {sphere }}=\sum_{\substack{j, \bar{\jmath} \in \mathrm{N}_{0} / 2 \\ j+\bar{\jmath} \in \mathrm{N}_{0}}}\left(V^{j} \otimes \bar{V}^{\bar{\jmath}}\right) \otimes\left(\mathcal{H}_{j^{2}}^{\mathrm{Vir}} \otimes \overline{\mathcal{H}}_{\bar{\jmath}^{2}}^{\mathrm{Vir}}\right) .
$$

As we have explained before, we can construct a (conformal) Ishibashi state (i.e. a state that satisfies (10) but not necessarily any additional gluing condition) for each tensor product $\mathcal{H}_{h}^{\mathrm{Vir}} \otimes \mathcal{H}_{\bar{h}}^{\mathrm{Vir}}$ for which the two Virasoro representations are conjugate, i.e. for which $h=\bar{h}$. Thus only the sectors with $j=\bar{\jmath}$ in the above decomposition give rise to Ishibashi states, and we obtain $V^{j} \otimes V^{j}$ conformal Ishibashi states for each $j \in \frac{1}{2} \mathbb{N}_{0}$. Let us denote these Ishibashi states by $|j ; m, n\rangle\rangle$, where $m$ and $n$ run from $-j,-j+1, \ldots, j-1, j$ and label the states in $V^{j}$. These Ishibashi states are thus labelled just like matrix elements of $\mathrm{su}(2)$ representations.

The most general conformal boundary conditions have not been known until recently 17] (we shall give the complete solution below), but a large class of $\mathrm{su}(2)$ preserving boundary states have been known for some time [36, 34]. They are believed to be consistent and fundamental since they can be obtained from the Cardy boundary states (that 
were shown to be consistent in [28, 29]) by marginal deformations. These boundary states are characterised by the gluing condition

$$
\left.\left.\left(\operatorname{Ad}_{(g \cdot \iota)}\left(J_{m}^{a}\right)+\bar{J}_{-m}^{a}\right) \| g\right\rangle\right\rangle=0 \quad \text { where } \quad \iota=\left(\begin{array}{cc}
0 & 1 \\
-1 & 0
\end{array}\right) .
$$

In terms of the conformal Ishibashi states they are given as

$$
\left.\| g\rangle\rangle=\frac{1}{2^{\frac{1}{4}}} \sum_{j, m, n} D_{m, n}^{j}(g)|j ; m, n\rangle\right\rangle,
$$

where $D_{m, n}^{j}(g)$ is the matrix element of $g$ in the representation $j$, and $\left.|j ; m, n\rangle\right\rangle$ denotes the Virasoro Ishibashi state labelled by the triple $(j ; m, n)$ as above. These boundary states include the Neumann and Dirichlet branes we have discussed before; in fact, the boundary state (47) agrees with (56) for

$$
g=\left(\begin{array}{cc}
0 & e^{i \frac{w}{2 \sqrt{2}}} \\
-e^{-i \frac{w}{2 \sqrt{2}}} & 0
\end{array}\right)
$$

while the Dirichlet brane (48) agrees with (56) for

$$
g=\left(\begin{array}{cc}
e^{i \frac{a}{\sqrt{2}}} & 0 \\
0 & e^{-i \frac{a}{\sqrt{2}}}
\end{array}\right)
$$

Provided that these boundary states are indeed consistent they have to satisfy the factorisation constraint, and we therefore have to have that

$$
D_{m_{1}, n_{1}}^{j_{1}}(g) D_{m_{2}, n_{2}}^{j_{2}}(g)=\sum_{j ; m, n} M_{j_{1} ; m_{1}, n_{1} j_{2} ; m_{2}, n_{2}}^{j ; m, n} D_{m, n}^{j}(g)
$$

where we have denoted the (rescaled) structure constant $2^{\frac{1}{4}} C \cdot F$ from (38) summarily by $M$. This equation has to hold for all $g \in \mathrm{SU}(2)$ (since we have a fundamental boundary condition for each such $g$ ). On the other hand, $M$ does not depend on $g$ since it is given in terms of the operator product coefficient $C$ and the fusing matrix $F$ that do not depend on any boundary conditions. Thus we can use the Peter-Weyl Theorem (see for example [37]) to deduce that this can only be the case if

$$
M_{j_{1} ; m_{1}, n_{1} j_{2} ; m_{2}, n_{2}}^{j ; m, n}=\left(j_{1} m_{1}, j_{2} m_{2} \mid j m\right)\left(j n \mid j_{1} n_{1}, j_{2} n_{2}\right)
$$

where $\left(j_{1} m_{1}, j_{2} m_{2} \mid j m\right)$ and $\left(j n \mid j_{1} n_{1}, j_{2} n_{2}\right)$ are the Clebsch-Gordan coefficients that describe the decomposition of the tensor product $j_{1} \otimes j_{2}$ in terms of the representation $j$. Indeed, the left hand side of $(59)$ is the matrix element between the states labelled by $\left(m_{1} \otimes m_{2}\right)$ and $\left(n_{1} \otimes n_{2}\right)$ in the tensor product of the representations $j_{1}$ and $j_{2}$; the Clebsch-Gordan coefficients describe the decomposition of this tensor product into irreducible representations, and therefore these matrix elements must agree with the right hand side of (59). Since the structure constants $M$ are uniquely determined by (59), we may conclude that $M$ must be given by (60).

Thus we have succeeded to determine the structure constants of the 'classifying algebra' (38) from the knowledge of a sufficiently large class of solutions. Given these structure constants we can now determine its most general solution; it is given by

$$
B^{(j ; m, n)}=\frac{1}{2^{\frac{1}{4}}} D_{m, n}^{j}(g) \quad \text { where } g \in \mathrm{SL}(2, \mathbb{C}) .
$$


Thus we can conclude that the most general fundamental conformal D-branes for this theory are (at most) labelled by group elements in $\mathrm{SL}(2, \mathbb{C})$ [17].

It is not difficult to check that all of these D-branes actually satisfy the Cardy condition (see [17] for a careful discussion of this issue). The D-branes that are associated to $\mathrm{SL}(2, \mathbb{C}) \backslash \mathrm{SU}(2)$ have complex couplings to some of the space-time fields, and may therefore be unphysical (at least from a string theory point of view). Apart from these unphysical branes, all conformal D-branes actually preserve the full $\mathrm{SU}(2)$ symmetry (since they satisfy the gluing condition (55)). This is quite surprising since the Virasoro condition is (on the face of it) much weaker, and one would have expected to find many more solutions.

\subsection{The conformal branes for other radii}

For $R \neq \frac{1}{\sqrt{2}}$ we can use similar techniques to determine all conformal D-branes of the theory, although the results are not as complete as for the self-dual case $R=\frac{1}{\sqrt{2}}$. The results depend on whether the radius of the circle is a ratio of the self-dual radius, or whether it is an irrational multiple of the self-dual radius. In the former case, i.e. if the radius is of the form

$$
R=\frac{M}{N} \frac{1}{\sqrt{2}},
$$

where $M$ and $N$ are coprime positive integers, the most general fundamental conformal D-branes can be described as follows [18]: every fundamental conformal D-brane is (i) either a Neumann or Dirichlet brane (i.e. has a boundary state given by (47) or (48), respectively); or (ii) it is a brane associated to an element in

$$
\mathrm{SU}(2) / \mathbb{Z}_{M} \times \mathbb{Z}_{N},
$$

that can be constructed by a formula similar to (56). (Details of the construction can be found in [18].) If we write an arbitrary group element of $\mathrm{SU}(2)$ as

$$
g=\left(\begin{array}{cc}
a & b \\
-b^{*} & a^{*}
\end{array}\right) \quad|a|^{2}+|b|^{2}=1,
$$

then the generator of $\mathbb{Z}_{N}$ acts as $a \mapsto e^{\frac{2 \pi i}{N}} a$, while the generator of $\mathbb{Z}_{M}$ acts as $b \mapsto e^{\frac{2 \pi i}{M}} b$. The branes associated to (63) are fundamental provided that $a b \neq 0$; on the other hand, for $a=0$ the brane associated to (64) is the superposition of $N$ Neumann branes (47) with evenly spaced Wilson lines, while for $b=0$, the brane described by (64) is the superposition of $M$ equidistantly spaced Dirichlet branes (48). The general D-branes in the family interpolate between these two extremal configurations. In fact, the Dirichlet or Neumann brane configurations merge into intermediate boundary states that can no longer be thought of as superpositions of fundamental branes. These intermediate branes are themselves fundamental, and do not preserve the $\mathrm{U}(1)$ symmetry.

The situation at an 'irrational' radius, i.e. if $\sqrt{2} R$ is irrational, can be formally deduced from the above by taking simultaneously $M, N \rightarrow \infty$. In this limit the branes labelled by (63) then only depend on the modulus of $a$ and $b$. Since $|a|^{2}+|b|^{2}=1$, there is therefore only one real parameter that we can take to be given by $x=2|a|^{2}-1$ with $-1 \leq x \leq 1$. In addition to the standard Neumann and Dirichlet branes the theory therefore has only an interval of branes labelled by $x$ [38, 39]. This interval of branes interpolates between a smeared Dirichlet brane (i.e. the integral of Dirichlet boundary states where we integrate over all possible positions on the circle) and a smeared Neumann brane (i.e. the integral of Neumann boundary states where we integrate over all possible Wilson lines on the dual circle). 


\subsection{Introducing fermions}

Up to now we have only discussed bosonic conformal field theories. For (world-sheet) fermions a few additional complications arise. First of all, we need an additional parameter $\eta= \pm 1$ that labels the different spin structures of the world-sheet. This parameter modifies the gluing condition of every world-sheet fermion. For example, the gluing condition for the superpartner $G$ of the stress-energy tensor is then

$$
\left.\left(G_{r}+i \eta \bar{G}_{-r}\right) \| B, \eta\right\rangle=0 .
$$

In the case with supersymmetry it is natural to consider only those boundary conditions that not only preserve the conformal symmetry (10) but are also superconformal, i.e. satisfy (65). Finally, we also need to make sure that the boundary states actually lie in the physical closed string spectrum, i.e. that they are GSO-invariant. In the onedimensional case, only one GSO-projection is consistent [40],

$$
P_{\mathrm{GSO}}^{0}=\frac{1}{2}\left(1+(-1)^{F+\bar{F}}\right),
$$

where $F$ and $\bar{F}$ are the left- and right-moving fermion number operators. This is to be contrasted with the more familiar GSO-projection

$$
P_{\mathrm{GSO}}=\frac{1}{4}\left(1+(-1)^{F}\right)\left(1 \pm(-1)^{\bar{F}}\right),
$$

that is relevant for the ten-dimensional superstring theories. The one-dimensional theory we are considering here is therefore the analogue of the type $0 \mathrm{~B}$ (or $0 \mathrm{~A}$ ) theory, rather than that of the type IIB (or IIA) theory [41, 42].

The analogue of the D-branes that preserve the full U(1) symmetry, i.e. that satisfy (42) or (43), satisfy now in addition gluing conditions for the superpartner $\psi$ of the current,

$$
\begin{aligned}
& \left.\left(\psi_{r}+i \eta \bar{\psi}_{-r}\right)|\mathrm{N}, \eta\rangle\right\rangle=0 \\
& \left.\left(\psi_{r}-i \eta \bar{\psi}_{-r}\right)|\mathrm{D}, \eta\rangle\right\rangle=0 .
\end{aligned}
$$

The most general such D-branes are conventional Neumann and Dirichlet branes that are then either the analogue of BPS branes (as in [43]) or the analogue of the non-BPS branes of Sen [7] (see also [44]). The construction of these D-branes will be briefly reviewed in the next section.

In addition to these $\mathrm{U}(1)$-preserving D-branes there are also superconformal D-branes, i.e. D-branes that only preserve (10) and (65) but not necessarily (42) and (68) or (43) and (69). If the radius of the circle is rational, $R=\frac{M}{N}$, where $M$ and $N$ are coprime positive integers, then these additional D-branes are associated to

$$
\mathrm{SU}(2) / \mathbb{Z}_{\tilde{M}} \times \mathbb{Z}_{N}
$$

where $\tilde{M}=2 M$ if $N$ is odd, and $\tilde{M}=M$ otherwise. These branes interpolate between brane anti-brane pairs and non-BPS branes [18] (see Figure 1). This is consistent with charge conservation since the overall R-R charge of both configurations vanishes.

\footnotetext{
${ }^{5}$ In this lecture we are restricting ourselves to the case with $N=1$ world-sheet supersymmetry. For higher supersymmetry, one should also preserve the additional supercurrents.
} 

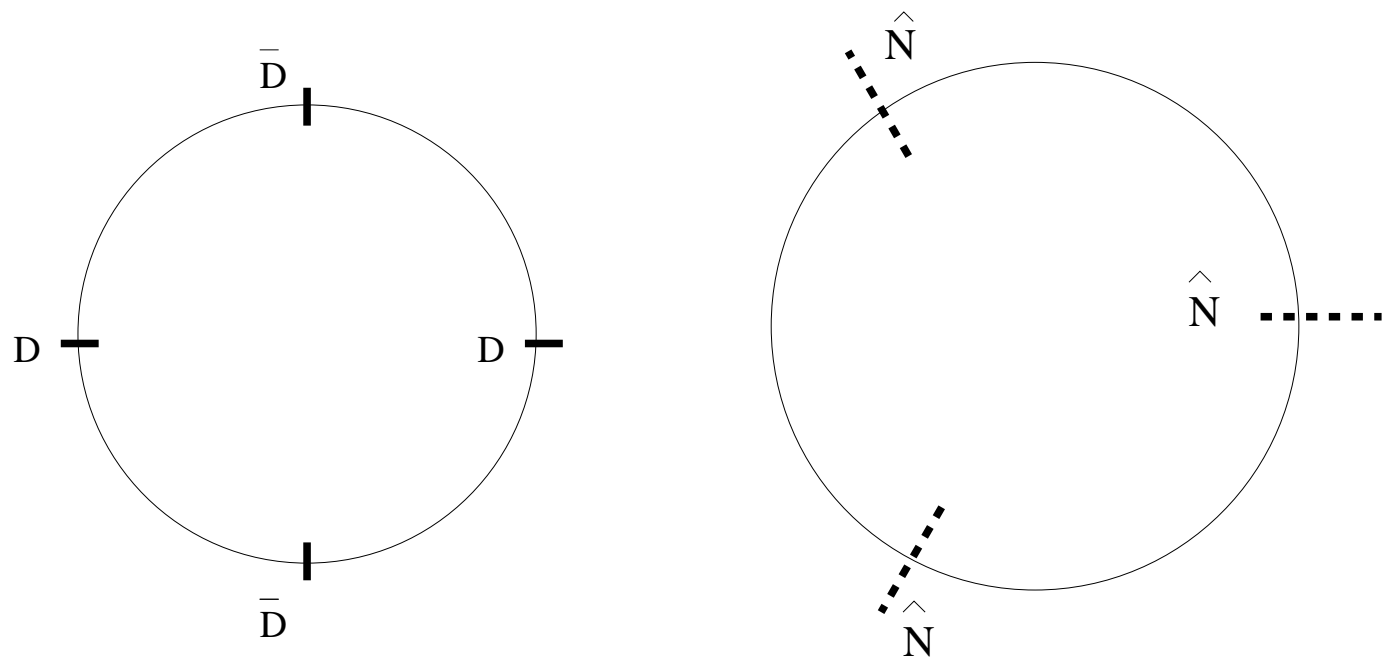

Figure 1: The brane configurations for $R=2 / 3$ corresponding to special group elements: on the left, $g$ is of the form (58) and the boundary state describes two Dirichlet branes $D$ and two anti-Dirichlet branes $\bar{D}$ distributed evenly over the target circle; on the right, $g$ is of the form (57), and we have three non-BPS Neumann branes $\hat{N}$ with Wilson lines that are evenly distributed over the dual circle.

\section{The ten-dimensional theories}

For the ten-dimensional string theories (that are ultimately of interest) the complete set of superconformal D-branes is not yet known. However, the branes that preserve the ten different $\mathrm{U}(1) \mathrm{s}$ can be easily described, and the restriction on the number of Neumann and Dirichlet directions for IIA/0A and IIB/0B can be straightforwardly understood. The relevant construction was reviewed in detail in [45]; we shall therefore be rather sketchy here, and only highlight some of the crucial features.

In the following we shall always work in light-cone gauge, and we shall only consider D-branes for which the boundary condition for both light-cone directions is Dirichlet. We are interested in constructing D-branes $\| \mathrm{Dp}\rangle\rangle$ for which $p+1$ of the ten directions satisfy a Neumann boundary conditions. Since we are working in light-cone gauge $p=-1, \ldots, 7$; the general case can be obtained from this analysis by a double Wick rotation [46].

Let us concentrate on the supersymmetric case in the following for which the GSO projection is (67). In this case the GSO-invariant D-branes are invariant under both $(-1)^{F}$ and $(-1)^{\bar{F}}$. Since

$$
(-1)^{\bar{F}}\left(\psi_{r}+i \eta \bar{\psi}_{-r}\right)=\left(\psi_{r}-i \eta \bar{\psi}_{-r}\right)(-1)^{\bar{F}}
$$

the GSO-invariant D-branes are necessarily superpositions of boundary states with both $\eta= \pm 1$. This fact is ultimately responsible for the emergence of fermions in the open string spectrum.

In the NS-NS sector, every non-trivial boundary state (for fixed $\eta$ ) can be made GSOinvariant (by adding to it a suitable boundary state with the opposite sign for $\eta$ ), but in the R-R sector this is not always possible. Indeed, in the R-R sector the boundary state must satisfy a gluing condition coming from the fermionic zero modes (i.e. from (68) 
and (69) with $r=0$ ), and this is not always compatible with the GSO-projection. As is explained in [45], the boundary state $\| \mathrm{Dp}\rangle\rangle_{\mathrm{R}-\mathrm{R}}$ is GSO-invariant provided

$$
p \text { is } \begin{cases}\text { even } & \text { for IIA } \\ \text { odd } & \text { for IIB. }\end{cases}
$$

As we have mentioned before, there are two types of U(1)-preserving branes that are known.

\subsection{Stable BPS branes}

The boundary states of the stable BPS D-branes are schematically given as

$$
\left.\left.\| \mathrm{Dp}\rangle\rangle=\| \mathrm{Dp}\rangle\rangle_{\mathrm{NS}-\mathrm{NS}} \pm i \| \mathrm{Dp}\right\rangle\right\rangle_{\mathrm{R}-\mathrm{R}}
$$

where $\| \mathrm{Dp}\rangle\rangle_{\mathrm{NS}-\mathrm{NS}}$ and $\left.\left.\| \mathrm{Dp}\right\rangle\right\rangle_{\mathrm{R}-\mathrm{R}}$ are suitably normalised superpositions of Ishibashi states in the NS-NS and the R-R sector, respectively. The sign between the NS-NS and the R-R component in (73) distinguishes between branes and anti-branes. These boundary states involve a R-R component, and they are therefore only GSO-invariant provided that $p$ satisfies (72). The D-branes satisfy Cardy's condition in that they lead to open strings of the form

$$
[\mathrm{NS}-\mathrm{R}] \frac{1}{2}\left(1+(-1)^{F}\right)
$$

i.e. to a total open string amplitude that is the trace over the NS-sector minus the trace over the R-sector, both with the insertion of the GSO-projector. Because of this GSOprojection, the tachyon from the open string NS-sector is removed, and the branes are stable.

\subsection{Unstable non-BPS branes}

The theory also possesses unstable non-BPS D-branes [7] whose boundary states are of the form [47]

$$
\| \mathrm{Dp}\rangle\rangle=\sqrt{2} \| \mathrm{Dp}\rangle\rangle_{\mathrm{NS}-\mathrm{NS}} .
$$

Here $\| \mathrm{Dp}\rangle\rangle_{\mathrm{NS}-\mathrm{NS}}$ is the same combination of Ishibashi states (including normalisation) that appeared in (73). As is explained in detail in [45], these branes occur for the complementary values of $p$ relative to (72). Their open string spectrum is now of the form

$$
[\mathrm{NS}-\mathrm{R}]
$$

without a GSO-projection. As a consequence the open string tachyon from the NS-sector is not removed, and the D-brane is unstable.

\subsection{Stable non-BPS branes}

As we have seen above, all stable (fundamental) U(1)-preserving D-branes of Type IIA/IIB string theory in flat space are actually BPS. However, this is not true in general. In particular, stable non-BPS D-branes exist for certain orbifold theories of Type IIA/IIB. The simplest example is the D0-brane of the orbifold of IIB on $T^{4} /(-1)^{F_{L}} I_{4}$ [48, 49]. Here

\footnotetext{
${ }^{6}$ Since the states in the open string R-sector correspond to spacetime fermions, they must contribute with a minus sign to this amplitude.
} 
$I_{4}$ denotes the inversion of the four torus directions, and $(-1)^{F_{L}}$ acts as \pm 1 on left-moving space-time fermions. The corresponding boundary state is schematically of the form

$$
\left.\left.\| \mathrm{D} 0\rangle\rangle=\| \mathrm{D} 0\rangle\rangle_{\mathrm{NS}-\mathrm{NS}} \pm i \| \mathrm{D} 0\right\rangle\right\rangle_{\mathrm{R}-\mathrm{R} ; \mathrm{T}},
$$

where the last component is in the twisted R-R sector. This boundary state is not BPS since it does not have a component in the untwisted R-R sector. However, it is nevertheless stable (at least for sufficiently large radii) since the corresponding open string is

$$
[\mathrm{NS}-\mathrm{R}] \frac{1}{2}\left(1+(-1)^{F} I_{4}\right) .
$$

This projection removes the zero-winding component of the tachyon in the open string NS-sector, and thus stabilizes the D-brane.

Stable non-BPS D-branes play an important role for understanding string dualities of supersymmetric string theories. For example the T-dual of the above D0-brane is a non-BPS D1-brane for IIA on $T^{4} / I_{4}$ [7, 国]. Since $T^{4} / I_{4}$ is an orbifold limit of K3, this theory is dual to the Heterotic string on $T^{4}$. The dual of the non-BPS D1-brane of the IIA theory can then be identified with a certain perturbative stable non-BPS state of the Heterotic theory [8]. This sheds some light on how string duality relates states that are not BPS, and that are therefore not protected from quantum corrections.

\section{Conclusions}

In this lecture I have described some of the key features in the construction of D-branes using conformal field theory techniques. As should have become apparent, this is a powerful construction that gives rise to exact string theoretic results.

I have described in detail the complete classification of the conformal D-branes for the simple case of a single free boson, and the supersymmetric analogue of a single boson and a single Majorana fermion. I have also sketched the more familiar construction of the standard U(1)-preserving D-branes for a number of ten-dimensional theories.

There are a number of avenues that it would be interesting to explore. In particular, it would be important to understand all superconformal D-branes for the ten-dimensional string theories. It would also be interesting to classify all the D-branes that preserve $N=2$ world-sheet supersymmetry. Indeed, most of the additional superconformal D-branes we have constructed are non-BPS and in fact unstable. In order to obtain space-time supersymmetric (stable) D-branes, one presumably needs to preserve $N=2$ supersymmetry on the world-sheet. Thus it would be interesting to see whether our analysis can be extended to that case.

\section{Acknowledgements}

I would like to thank the organisers for organising a very stimulating and interesting school, and for giving me the opportunity to present these lectures. I thank Andreas Recknagel and Gérard Watts for collaborations and useful discussions on many issues discussed in these lectures. I am also grateful to Hanno Klemm and Andreas Recknagel for a careful reading of a draft of these notes.

I am grateful to the Royal Society for a University Research Fellowship. This work is also partly supported by EU contract HPRN-CT-2000-00122, and the PPARC special grant "String Theory and Realistic Field Theory", PPA/G/S/1998/0061. 


\section{References}

[1] G. Moore and R. Minasian, J. High Energy Phys. 9711 (1997) 002 hep-th/9710230.

[2] E. Witten, J. High Energy Phys. 9812 (1998) 019 hep-th/9810188.

[3] M. Douglas, D-branes, categories and $N=1$ supersymmetry hep-th/0011017.

[4] M.R. Douglas and G. Moore, D-branes, quivers, and ALE instantons hep-th 9603167].

[5] M. Frau, I. Pesando, S. Sciuto, A. Lerda and R. Russo, Phys. Lett. B400 (1997) 52 hep-th/9702037.

[6] P. Di Vecchia, M. Frau, I. Pesando, S. Sciuto, A. Lerda and R. Russo, Nucl. Phys. B507 (1997) 259 hep-th/9707068.

[7] A. Sen, J. High Energy Phys. 9812 (1998) 021 hep-th/9812031.

[8] O. Bergman and M.R. Gaberdiel, J. High Energy Phys. 9903 (1999) 013 hep th/9901014.

[9] D-E. Diaconescu, M.R. Douglas and J. Gomis, J. High Energy Phys. 9802 (1998) 013 [hep-th/9712230.

[10] D-E. Diaconescu and J. Gomis, J. High Energy Phys. 0010 (2000) 001 hepth/9906242].

[11] M.R. Gaberdiel and A. Sen, J. High Energy Phys. 9911 (1999) 008 hep-th/9908060.

[12] M.R. Gaberdiel and B. Stefański, Nucl. Phys. B578 (2000) 58 [hep-th/9910109].

[13] A. Recknagel and V. Schomerus, Nucl. Phys. B531 (1998) 185 [hep-th/9712186].

[14] M. Gutperle and Y. Satoh, Nucl. Phys. B555 (1999) 477 hep-th/9902120].

[15] J. Fuchs, C. Schweigert and J. Walcher, Nucl. Phys. B588 (2000) 110 hepth/0003298].

[16] I. Brunner, M.R. Douglas, A. Lawrence and Ch. Römelsberger, J. High Energy Phys. 0008 (2000) 015 hep-th/9906200.

[17] M.R. Gaberdiel, A. Recknagel and G.M.T. Watts, The conformal boundary states for $S U(2)$ at level 1 [hep-th/0108102].

[18] M.R. Gaberdiel and A. Recknagel, J. High Energy Phys. 0111 (2001) 016 hepth/0108238].

[19] C. Vafa, Phys. Lett. B199 (1987) 195.

[20] H. Sonoda, Nucl. Phys. B311 (1989) 401.

[21] G. Moore and N. Seiberg, Commun. Math. Phys. 123 (1989) 177.

[22] D.C. Lewellen, Nucl. Phys. B372 (1992) 654.

[23] J.L. Cardy and D.C. Lewellen, Phys. Lett. B259 (1991) 274. 
[24] G. Pradisi, A. Sagnotti and Ya. S. Stanev, Phys. Lett. B381 (1996) 97 hepth/9603097.

[25] I. Runkel, Nucl. Phys. B549 (1999) 563 hep-th/9811178.

[26] R.E. Behrend, P.A. Pearce, V.B. Petkova and J.-B. Zuber, Nucl. Phys. B579 (2000) 707 hep-th/9908036.

[27] I. Runkel, Nucl. Phys. B579 (2000) 561 hep-th/9908046.

[28] G. Felder, J. Fröhlich, J. Fuchs and C. Schweigert, Phys. Rev. Lett. 84 (2000) 1659 hep-th/9909140.

[29] G. Felder, J. Fröhlich, J. Fuchs and C. Schweigert, Correlation functions and boundary conditions in RCFT and three-dimensional topology hep-th/9912239.

[30] T. Gannon, Boundary conformal field theory and fusion ring representations hepth/0106105.

[31] N. Ishibashi, Mod. Phys. Lett. A4 (1989) 251.

[32] J.L. Cardy, Nucl. Phys. B324 (1989) 581.

[33] E. Verlinde, Nucl. Phys. B300 (1988) 360.

[34] A. Recknagel and V. Schomerus, Nucl. Phys. B545 (1999) 233 [hep-th/9811237.

[35] J. Fuchs and C. Schweigert, Phys. Lett. B414 (1997) 251 (1997) hep-th/9708141.

[36] M.B. Green and M. Gutperle, Nucl. Phys. B460 (1996) 77 hep-th/9509171.

[37] A.W. Knapp, Representation theory of semisimple groups: an overview based on examples, Princeton University Press, Princeton (1986).

[38] D. Friedan, The space of conformal boundary conditions for the $c=1$ Gaussian model, unpublished note (1999).

[39] R.A. Janik, Nucl. Phys. B618 (2001) 675 hep-th/0109021.

[40] L.J. Dixon, P. Ginsparg and J.A. Harvey, Nucl. Phys. B306 (1988) 470.

[41] L.J. Dixon and J.A. Harvey, Nucl. Phys. B274 (1986) 93.

[42] N. Seiberg and E. Witten, Nucl. Phys. B276 (1986) 272.

[43] O. Bergman and M.R. Gaberdiel, Nucl. Phys. B499 (1997) 183 [hep-th/9701137.

[44] D.M. Thompson, Descent relations in type OA/0B [hep-th/0105314.

[45] M.R. Gaberdiel, Class. Quant. Grav. 17 (2000) 3483 hep-th/0005029.

[46] M.B. Green and M. Gutperle, Nucl. Phys. B476 (1996) 484 hep-th/9604091.

[47] P. Hořava, Adv. Theor. Math. Phys. 2 (1998) 1373 hep-th/9812135.

[48] O. Bergman and M.R. Gaberdiel, Phys. Lett. B441 (1998) 133 hep-th/9806155.

[49] A. Sen, J. High Energy Phys. 9808 (1998) 010 hep-th/9805019. 